\title{
Ocorrência e Sazonalidade de Muscóides (Diptera, Calliphoridae) de Importância Sanitária no Município de Itaboraí, RJ, Brasil
}

\author{
José Antonio Batista-da-Silva ${ }^{\circledR}$, Gonzalo Efrain Moya-Borja² \& Margareth Maria de Carvalho Queiroz \\ 1. Laboratório de Transmissores de Leishmaniose (Setor de Entomologia Médica e Forense) do Instituto Oswaldo Cruz-IOC/FIOCRUZ e-mail: \\ zeize@uol.com.br (Autor para correspondência ${ }^{\bowtie}$ ), e-mail: mmcqueiroz@uol.com.br. 2. Universidade Federal Rural do Rio de Janeiro - Instituto de \\ Biologia, e-mail: gemoya@ufrrj.br.
}

EntomoBrasilis 3 (1): 16-21 (2010)

Resumo. Este trabalho teve como objetivo contribuir com o conhecimento da entomofauna de Calliphoridae (Diptera) no município de Itaboraí, RJ, Brasil e quantificar as espécies mais predominantes de importância sanitária. As moscas foram capturadas em oito diferentes pontos no período de um ano, usando sempre isca de peixe. Após triagem, as espécies foram separadas por espécie e inseridas na coleção entomológica do Laboratório de Transmissores de Leishmaniose (Setor de Entomologia Médica e Forense) do Instituto Oswaldo Cruz - IOC/FIOCRUZ. Foram capturadas 1792 moscas pertencentes a sete (7) espécies da família Calliphoridae: Chloroprocta idioidea (Robineau-Desvoidy) (0,11\%), Chrysomya megacephala (Fabricius) (87,94\%), Chrysomya albiceps (Wiedemann) (6,70\%), Chrysomya putoria (Wiedemann) (1,23\%), Cochliomyia macellaria (Fabricius) (o,56\%), Hemilucilia segmentaria (Fabricius) (o,33\%), Lucilia eximia (Wiedemann) (3,13\%).

Palavras-chave: Calliphoridae, importância médica e veterinária, moscas, Itaboraí

\section{Occurrence and seasonality of muscoid (Diptera, Calliphoridae) of public health importance in Itaboraí (RJ), Brazil}

Abstract. This work was carried out to contribute to the knowledge of Calliphoridae flies (Diptera) in Itaboraí, RJ, Brazil and quantify the predominant species of health importance. The flies were captured in eight different points in the city over a one year period, always using fish as bait, separated by species and kept properly in an entomological box in the Laboratório de Transmissores de Leishmaniose (Setor de Entomologia Médica e Forense) - IOC / FIOCRUZ, RJ. A total of 1792 Calliphoridae flies were captured, belonging to seven (7) species: Chloroprocta idioidea (Robineau-Desvoidy) (0.11\%), Chrysomya megacephala (Fabricius) (87.94\%), Chrysomya albiceps (Wiedemann) (6.70\%), Chrysomya putoria (Wiedemann) (1.23\%), Cochliomyia macellaria (Fabricius) (0.56\%), Hemilucilia segmentaria (Fabricius) (0.33\%), Lucilia eximia (Wiedemann) (3.13\%).

Keywords: Calliphoridae, fly, Itaboraí, medical and veterinary importance

A família Calliphoridae é de grande importância na área médica e veterinária, pois algumas espécies sinantrópicas estão ligadas a transmissão de ovos de helmintos, oocistos de protozoários, bactérias, vírus, fungos (QUEIROZ et al. 2005) e ainda produzem miĺases tanto homens quanto em animais domésticos em centro urbanos e suas periferias (BATISTADA SILVA et al. 2009). Esta família pertence à ordem Diptera, subordem Brachycera, divisão Cyclorrapha, e está amplamente distribuída em toda região Neotropical, sendo o estudo ecológico das mesmas de grande importância no controle da disseminação de agentes patogênicos de diversas doenças.

Além da importância médica dos muscóides, a importância forense vem se destacando nos países desenvolvidos, onde o conhecimento da biologia de algumas espécies como Lucilia eximia (Wiedemann), Hemilucilia segmentaria (Fabricius) Cochliomyia macellaria (Fabricius) e Chrysomya megacephala (Fabricius), são importantes na datação do intervalo pós morte (SALVIANO et al. 1996).

O estabelecimento de espécies do gênero Chrysomya no novo Mundo, provenientes da África (IMBIRIBA et al. 1977; GUIMARÃES et al. 1978 e 1979), têm afetado muscóides da fauna nativa, provocando o seu deslocamento ou extinção em determinados nichos, e ainda provocando a fuga de larvas de outras espécies pela liberação de alomônios por larvas de Chrysomya albiceps (AGUIAR-COELHO et al. 1995).
O levantamento dos dípteros califorídeos no município de Itaboraí se destinou a identificar as espécies existentes na área, quantificar as predominantes, destacando as proporções de machos e fêmeas, e ainda analisar a influência de fatores abióticos tais como: temperatura, umidade do ar e pluviosidade, e ainda proporcionar através do presente estudo, identificar as estações de ocorrências desta família no mesmo município.

\section{MATERIAL E MÉTODOS}

Todos os espécimes foram coletados no período de março de 2007 a fevereiro de 2008 no município de Itaboraí.

Foi realizada uma coleta quinzenal, totalizando 24 coletas durante um ano. Durante as capturas, foram utilizadas oito armadilhas confeccionadas em recipiente plástico com $35 \mathrm{~cm}$ de altura e $15 \mathrm{~cm}$ de diâmetro. Na parte inferior, revestida por material plástico preto, foram feitas quatro aberturas. A parte superior foi separada da inferior por um funil plástico, para permitir que o inseto que alcançasse o fundo do recipiente para abordagem da isca, pudesse passar para a parte superior, ficando impedido de voltar.

Foram montadas quatro armadilhas nas coordenadas $\mathrm{S}$ 220 43' 27" W 420 57' 53" e quatro nas coordenadas, S 22044 ' 52 " e W 420 55' 29" a uma distância aproximada de 100m uma da outra em posições aleatórias, mantendo-se sempre a distância padrão. 
As armadilhas foram suspensas a uma altura de $1,20 \mathrm{~m}$ do solo contendo $100 \mathrm{~g}$ de isca de peixe (sardinha) em decomposição.

Após cada captura, todos os espécimes foram acondicionados em potes plásticos, contendo etanol a $70 \%$. Levados ao Laboratório de Transmissores de Leishmaniose (Setor de Entomologia Médica e Forense) do Instituto Oswaldo Cruz - IOC/FIOCRUZ, onde os potes foram separados por data de coleta e seu conteúdo foi separado, identificado e contado, logo após alguns exemplares de cada espécie foram devidamente alfinetados einseridos na coleção permanente dolaboratório. Para tal procedimento, utilizou-se um microscópio estereoscópico e chaves dicotômicas para a identificação da família e das espécies de acordo com MELLO (2003).

\section{RESULTADOS}

Durante este trabalho foram capturadas 1792 moscas pertencentes a sete (7) espécies da família Calliphoridae: Chloroprocta idioidea (Robineau-Desvoidy), Chrysomya megacephala (Fabricius), Chrysomya albiceps (Wiedemann), Chrysomya putoria (Wiedemann), Cochliomyia macellaria (Fabricius), Hemilucilia segmentaria (Fabricius) e Lucilia eximia (Wiedemann).

A Tabela 1 apresenta as freqüências das espécies em cada estação do ano e a Figura 1 apresenta a demonstração gráfica do número de indivíduos da família Calliphoridae capturados em cada estação do ano. C. megacephala (Figura o2) foi a espécie mais predominante em todas as estações, mas sendo mais abundante principalmente na primavera. C. albiceps (Figura 02) e C. putoria (Figura 02) também apresentaram maior predominância na primavera, reduzindo sua presença nas outras estações do ano.

Verificou-se que C. macellaria foi pouco capturada, apresentando somente dez (10) espécimes identificados, com baixa freqüência na primavera $(0,16 \%)$, no verão $(1,12 \%)$ e outono (4,76\%), não sendo encontrada no inverno. C. idioidea foi a espécie menos capturada, apresentando apenas dois (2) indivíduos no verão, já $L$. eximia foi encontrada nas quatro estações do ano, mas revelando maior presença no verão e no outono. A segunda espécie menos capturada foi $H$. segmentaria, com apenas seis (6) indivíduos no verão (Figura 3).

As estações do ano representam um bom indicador de estudo populacional, pois se pode observar na Tabela 1 e na Figura 1 que as variações ocorrem fortemente a cada estação. Ainda de acordo com a Tabela 1, pode-se notar que a única estação onde houve ocorrência de todas as sete espécies capturadas foi o verão, revelando a estação como maior freqüência de todas as sete espécies, simultaneamente.

O inverno foi a estação com menor número de capturas de indivíduos da família Calliphoridae, com 64 (3,57\%) do total de indivíduos, seguido do outono com 84 (4,68\%) (Figura 1). A estação onde houve maior ocorrência de califorídeos foi a primavera com 1288 ou $71,88 \%$ de todos os indivíduos capturados, seguido do verão com 356 (19,87\%) (Figura 1).

A Figura 4 apresenta as ocorrências da família Calliphoridae quanto à temperatura e a umidade.

A espécie C. megacephala revelou maior ocorrência em $31^{\circ} \mathrm{C}$ de temperatura e umidade $62 \%$, mas como se pode observar, esta espécie foi capturada em temperaturas compreendidas entre $21^{\circ} \mathrm{C}$ a $38^{\circ} \mathrm{C}$ e umidade entre $47 \%$ a $87 \%$. C. albiceps foi mais restritiva, variando sua preferência entre $26^{\circ} \mathrm{C}$ até $31^{\circ} \mathrm{C}$, com umidade de $47 \%$ até $87 \%$ e preferência por umidade de $62 \%$. C. putoria apresentou preferência por temperaturas mais altas, de $31^{\circ} \mathrm{C}$ até $38^{\circ} \mathrm{C}$, e umidade compreendida entre $47 \%$ a $87 \%$, sendo $62 \%$ a umidade ideal. As três espécies do gênero Chrysomya apresentaram semelhança com relação a estas duas variáveis abióticas, indicando uma grande capacidade adaptativa destas espécies exóticas.

Tabela 1: Número total e individual de moscas da família Calliphoridae capturadas e seus respectivos percentuais por espécie, distribuídos nas quatro estações do ano, no período de março 2007 a fevereiro de 2008 no município de Itaboraí, RJ, Brasil.

\begin{tabular}{|c|c|c|c|c|c|c|c|c|c|c|}
\hline \multirow{2}{*}{ Espécies } & \multicolumn{2}{|c|}{ Primavera } & \multicolumn{2}{|c|}{ Verão } & \multicolumn{2}{|c|}{ Outono } & \multicolumn{2}{|c|}{ Inverno } & \multicolumn{2}{|c|}{ Total } \\
\hline & No & $\%$ & No & $\%$ & No & $\%$ & No & $\%$ & No & $\%$ \\
\hline Chrysomya megacephala & 1198 & 93,01 & 294 & 82,58 & 40 & 47,62 & 44 & 68,75 & 1576 & 87,94 \\
\hline Chrysomya albiceps & 66 & 5,12 & 24 & 6,74 & 16 & 19,05 & 14 & 21,88 & 120 & 6,70 \\
\hline Chrysomya putoria & 20 & 1,55 & 02 & 0,56 & oo & 0,00 & oo & 0,00 & 22 & 1,23 \\
\hline Cochliomyia macellaria & 02 & 0,16 & 04 & 1,12 & 04 & 4,76 & oo & 0,00 & 10 & 0,56 \\
\hline Chloroprocta idioidea & oo & 0,00 & 02 & 0,56 & oo & 0,00 & oo & 0,00 & 02 & 0,11 \\
\hline Lucilia eximia & 02 & 0,16 & 24 & 6,74 & 24 & 28,57 & 06 & 9,38 & 56 & 3,13 \\
\hline Hemilucilia segmentaria & oo & 0,00 & 06 & 1,69 & oo & 0,00 & oo & 0,00 & 06 & 0,33 \\
\hline Total & 1288 & 100 & 356 & 100 & 84 & 100 & 64 & 100 & 1792 & 100 \\
\hline
\end{tabular}

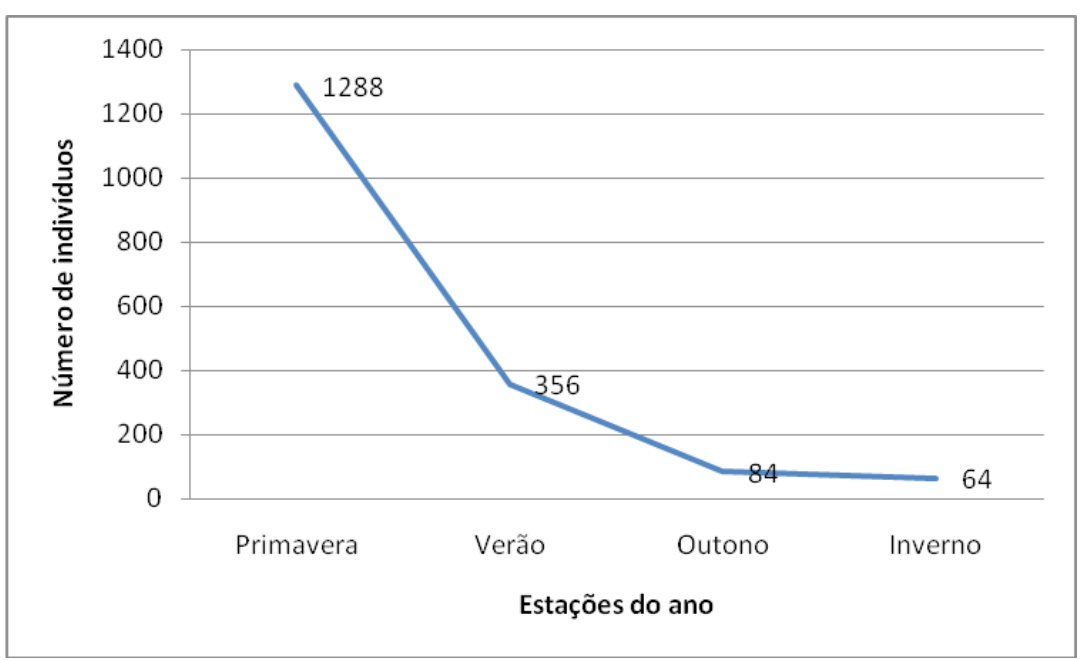

Figura 1. Demonstração gráfica do número de indivíduos da família Calliphoridae capturados em cada estação do ano, no município de Itaboraí, RJ, Brasil. 


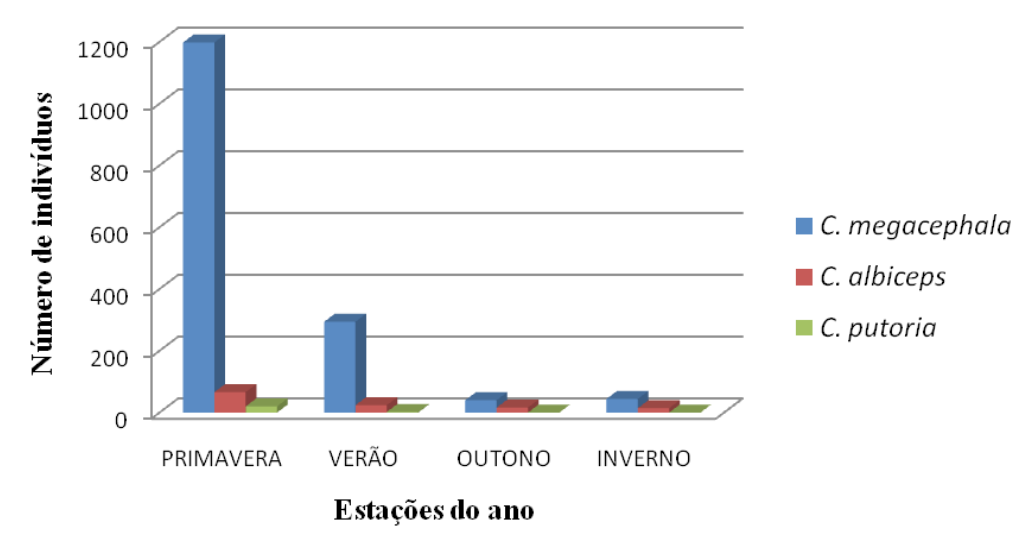

Figura 2. Distribuição numérica de Chrysomya megacephala, Chrysomya albiceps e Chrysomya putoria nas quatro estações do ano no município de Itaboraí, RJ, Brasil.

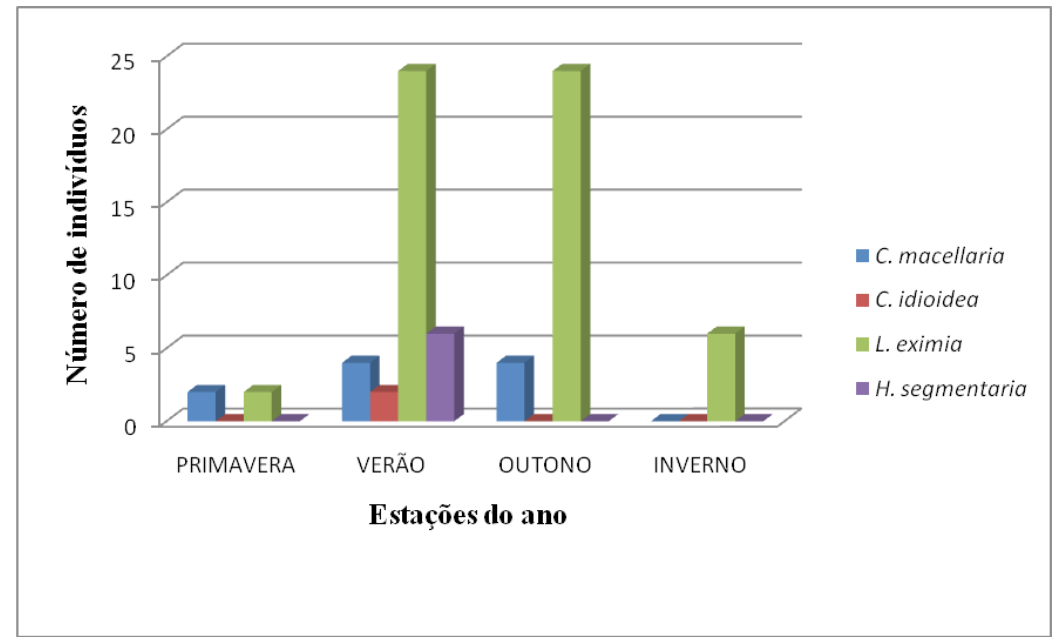

Figura 3. Distribuição numérica de Cochliomyia macellaria, Chloroprocta idioidea, Lucilia eximia e Hemilucilia segmentaria nas quatro estações do ano no município de Itaboraí, RJ, Brasil.

O díptero C. macellaria, apesar de ter apresentado ampla ocorrência quanto à temperatura, $21^{\circ} \mathrm{C}$ até $31^{\circ} \mathrm{C}$, demonstrou maior preferência a valores próximos de $31^{\circ} \mathrm{C}$, mantendo a preferência por umidade ideal de $62 \%$, como as espécies relatadas anteriormente.

As espécies $C$. idioidea e $H$. segmentaria apresentaram as mesmas preferências quanto a temperatura, $26^{\circ} \mathrm{C}$ e quanto a umidade, $87 \%$.

Lucilia eximia foi encontrada em temperaturas variando entre $21^{\circ} \mathrm{C}$ a $34^{\circ} \mathrm{C}$, preferindo aquelas entre $24^{\circ} \mathrm{C}$ até $26^{\circ} \mathrm{C}$. A umidade de ocorrência desta espécie ficou entre 52\% a $88 \%$, sendo a umidade ideal de $87 \%$, o que revela grande adaptação às baixas quanto às altas umidades.

Durante todas as coletas foram registrados os valores acumulados, até a respectiva data, mensalmente, para as precipitações pluviométricas (INPE 2008)

Foi observada maior ocorrência da família Calliphoridae a precipitações compreendidas entre $316 \mathrm{~mm}$ a $360 \mathrm{~mm}$ com um percentual de $68,19 \%$ de todos os indivíduos capturados.

A segunda maior ocorrência foi entre o $\mathrm{mm}$ e $45 \mathrm{~mm}$, com 21,88\%,

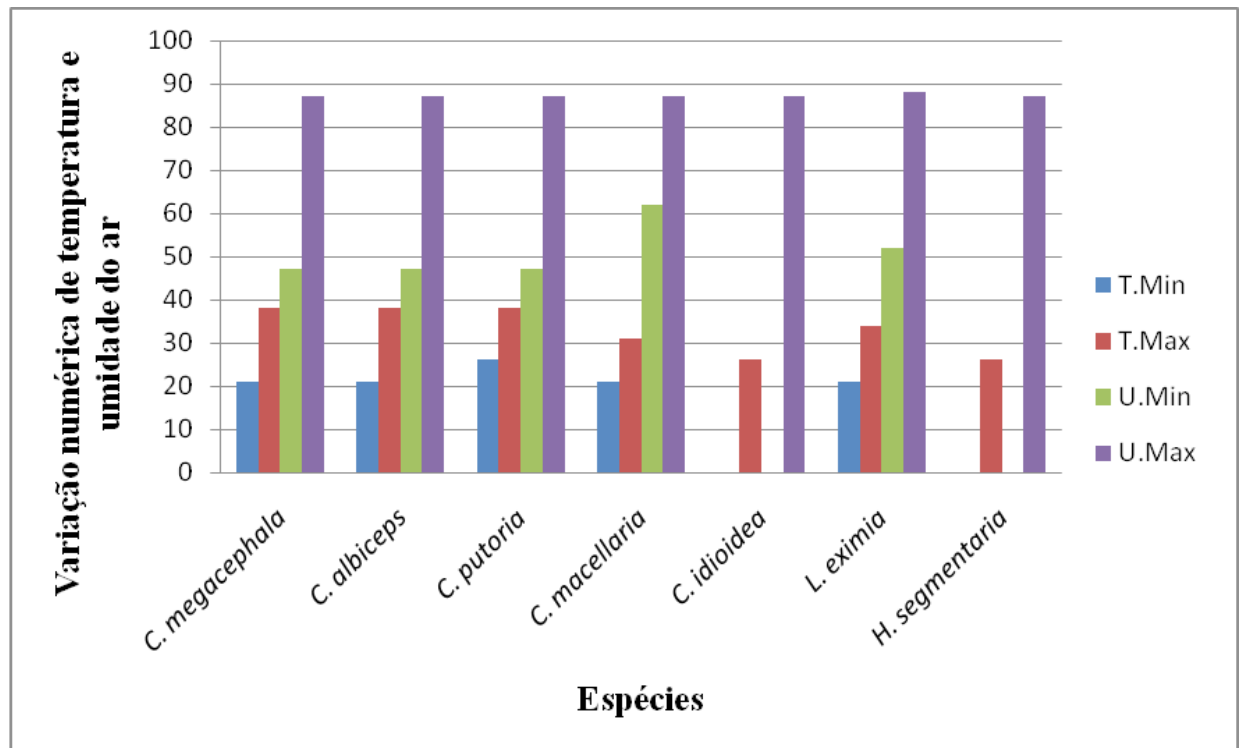

Figura 4. Demonstração gráfica das temperaturas e umidades mínimas e máximas de ocorrência de sete espécies capturadas no município de Itaboraí, RJ, Brasil. 
um número menor de indivíduos capturados (Tabela 2).

Não foram registrados valores pluviométricos compreendidos entre $91 \mathrm{~mm}$ e $135 \mathrm{~mm}, 226 \mathrm{~mm}$ e $270 \mathrm{~mm}, 361$ $\mathrm{mm}$ e $585 \mathrm{~mm}$, assim como entre $631 \mathrm{~mm}$ e $720 \mathrm{~mm}$.

Tabela 2. Valores das precipitações pluviométricas em mm, acumulados até cada data de coleta em cada mês, distribuídos em classes com seus referidos valores numéricos e os percentuais obtidos, para a família Calliphoridae no município de Itaboraí, RJ, Brasil.

\begin{tabular}{|c|c|c|}
\hline \multirow{2}{*}{$\begin{array}{l}\text { Precipitação } \\
\text { (mm) }\end{array}$} & \multicolumn{2}{|c|}{ Valores para a família } \\
\hline & $\mathbf{N}$ & $\%$ \\
\hline $0-45$ & 392 & 21,88 \\
\hline $46-90$ & 44 & 2,46 \\
\hline $91-135$ & - & - \\
\hline $136-180$ & 18 & 1,00 \\
\hline $181-225$ & 20 & 1,12 \\
\hline $226-270$ & - & - \\
\hline $271-315$ & 26 & 1,45 \\
\hline $316-360$ & 1222 & 68,19 \\
\hline $361-405$ & - & - \\
\hline $406-450$ & - & - \\
\hline $451-495$ & - & - \\
\hline $496-540$ & - & - \\
\hline $541-585$ & - & - \\
\hline $586-630$ & 50 & 2,79 \\
\hline $631-675$ & - & - \\
\hline $676-720$ & - & - \\
\hline $721-765$ & 20 & 1,12 \\
\hline Total & 1792 & 100 \\
\hline
\end{tabular}

\section{DISCUSSÃO}

Durante doze meses foram observados padrões de comportamento de sete espécies da família Calliphoridae no município de Itaboraí. A ocorrência de cada espécie em relação à estação do ano, pluviosidade, assim como as temperaturas e umidades relativas do ar locais foram observadas e comparadas entre si.

Verifica-se que a espécie $C$. megacephala foi melhor adaptada, podendo ser encontrada nas quatro estações do ano, principalmente na primavera e no verão, confirmando assim os dados de d'ALMEIDA \& ALMEIDA (1998). O número crescente desta espécie vem sendo constatado, fato que pode ser confirmado por OLIVEIRA et al. (1999), no Jardim Zoológico do Rio de Janeiro, onde o número total de espécimes do gênero Chrysomya capturado, alcançou $91 \%$.

Ao apresentar relativa adaptação as variações térmicas $\left(21^{\circ} \mathrm{C}\right.$ até $\left.38^{\circ} \mathrm{C}\right)$ e capacidade de se manter em atividade em umidades compreendidas entre $47 \%$ até $87 \%$, revelam sua importância médico-veterinária, ecológica e forense, dentre os principais muscóides aqui estudados, visto que estes fatores abióticos são ótimos para o desenvolvimento de microrganismos patogênicos, assim como decompositores.

A segunda espécie com maior número de capturas foi C. albiceps A presença deste muscóide se fez em um percentual bastante elevado no total de coletas em observações feitas por FERREIRA et al. (1995) e SANTOS et al. (1996), em Goiânia, Goiás, por OLIVEIRA et al. (1999) no Jardim Zoológico da cidade do Rio de Janeiro, corroborando as observações feitas neste trabalho. Semelhante a $C$. megacephala, $C$. albiceps também revelou preferência pela primavera e pelo verão, sendo capturada nas quatro estações do ano, sob as mesmas condições de temperatura e umidade.

A espécie C. putoria se alimenta em diversos tipos de materiais orgânicos em decomposição, de acordo com GREENBERG (1971) e d'ALMEIDA \& ALMEIDA (1998), principalmente carnes e raramente em fezes, podendo ser um potencial vetor de bactérias enteropatogênicas e enterovírus como citada por FURLANETTO et al. (1984). Esta espécie dentre o gênero Chrysomya, revelou-se como a espécie menos freqüente, apresentando preferência por temperaturas mais elevadas, $31^{\circ} \mathrm{C}$ até $38^{\circ} \mathrm{C}$, o que possivelmente reflita a baixa freqüência desta espécie.

A isca de peixe assim como outros fatores ambientais como, por exemplo, a precipitação pluviométrica podem ter também interferido na captura e na população desta espécie, fato este observado por BAUMGARTNER \& GREENBERG (1985), no Peru e por FERREIRA et al. (1995) em Goiânia na área rural.

De acordo com MADEIRA et al. (1982), C. macellaria em Belo Horizonte foi considerada a terceira espécie mais abundante, de maio a setembro de 1978. Esta espécie foi citada por LINHARES (1981) como sendo uma das mais abundantes e mais associadas com o homem, e segundo SALVIANO et al. (1996), pode ocorrer com bastante freqüência nos cadáveres em decomposição.

No Rio de Janeiro, d'ALMEIDA \& LOPES (1983) consideraram $C$. macellaria, como uma espécie quase exclusiva de área rural, assim como FERREIRA (1983) em Goiânia, Goiás e FERREIRA \& BARBOLA (1998), em Curitiba, Paraná. No Peru, de acordo com BAUMGARTNER \& GREENBERG (1985), esta espécie é encontrada a altitudes abaixo de $2450 \mathrm{~m}$ e ainda esses autores sugeriram que a umidade e a precipitação não apresentaram importância na sua distribuição.

Além da forte competição citada por d'ALMEIDA \& LOPES (1983), AGUIAR-COELHO \& MILWARD-DE-AZEVEDO (1995 e 1998) e d'ALMEIDA et al. (1997), com as espécies do gênero Chrysomya, espécies exóticas introduzidas no Brasil por refugiados portugueses vindos da África, PRADO \& GUIMARÃES (1982) chamaram a atenção para a alta capacidade de adaptação e dispersão do gênero Chrysomya na região Neotropical, o que pode ter influenciado no baixo percentual de $C$. macellaria encontrado.

A terceira espécie menos capturada foi C. macellaria , podendo ser observada na primavera, no verão e no outono, não sendo encontrada no inverno. Quanto à temperatura preferida por esta espécie, dentre os poucos exemplares capturados, pode-se detectar aquelas compreendidas entre $21^{\circ} \mathrm{C}$ até $31^{\circ} \mathrm{C}$. A umidade do ar, apesar de ampla, $62 \%$ até $87 \%$, não pareceu ser um fator isolado para a baixa freqüência desta espécie.

A espécie $C$. idioidea foi encontrada por d'ALMEIDA \& LOPES (1983), no Rio de Janeiro, em área rural, urbana e florestal, sendo muito mais abundante na área florestal, com 76,82\%. FERREIRA (1978), em Curitiba e LINHARES (1981), em Campinas, não registraram sua presença, já BAUMGARTNER \& GREENBERG (1985) registraram sua presença, no Peru, em área florestal, sendo atraída por isca de peixe e até mesmo por frutos.

Desta forma, $C$. idioidea, pode ser considerada uma espécie tipicamente florestal e bem adaptada as quatro estações do ano, mas dando preferência as estações mais frias. Seu substrato de preferência não foi aqui bem evidenciado, visto que o único utilizado foi à base de peixe, proporcionando um percentual difícil de ser analisado, mas acredita-se que sua preferência sejam frutos em decomposição ou até mesmo fezes de animais, pois os mesmos podem ser encontrados em maior abundância na floresta.

De acordo com FERREIRA \& BARBOLA (1998), $H$. segmentaria só apresentou preferência por área florestada na região metropolitana de Curitiba. Segundo OLIVEIRA et al. (1999), no Jardim Zoológico do Rio de Janeiro, que é uma região metropolitana com ampla área de cobertura vegetal, sua freqüência maior ocorreu no inverno.

BAUMGARTNER \& GREENBERG (1985) na Costa Rica, relataram que esta espécie tem distribuição irregular nas montanhas e baixa distribuição nas florestas tropicais.

As duas espécies menos capturadas foram $C$. idioidea e 
H. segmentaria, sendo o verão a única estação preferida, assim consideradas espécies acidentais. A temperatura de $26^{\circ} \mathrm{C}$ e a umidade do ar $87 \%$ foram às preferidas por ambas as espécies.

De acordo com FERREIRA (1978), em Goiânia, Goiás, L. eximia foi à espécie mais freqüente no verão, sendo também observada nas demais estações, atraída por fígado, fezes e sardinha.

LINHARES (1981) considerou esta espécie pouco abundante em Campinas, São Paulo, com maior prevalência na área rural, considerando-a assim hemissinantrópica. Pode-se considerar, mediante resultados obtidos por MADEIRA et al. (1982), em Belo Horizonte, Minas Gerais e FERREIRA (1983), em Goiânia, Goiás, que esta espécie apresenta preferência por ambientes rurais habitados e, de acordo com d'ALMEIDA \& LOPES (1983), no Rio de Janeiro, que citaram a presença desta espécie em ambientes urbanos, uma espécie de hábitos sinantrópicos. Segundo BAUMGARTNER \& GREENBERG (1985), esta espécie apresenta-se amplamente distribuída na região neotropical e neártica.

A freqüência de $L$. eximia parece se manter elevada quando atraída por isca de fígado como cita d'ALMEIDA (1994), na floresta da Tijuca e por suco de laranja com açúcar em área rural, de acordo com SANTOS et al. (1996), Goiânia, Goiás.

L. eximia foi capturada nas quatro estações, mas demonstrou preferência pelo verão e pelo outono. As temperaturas preferidas desta espécie ficaram entre $24^{\circ} \mathrm{C}$ até $26^{\circ} \mathrm{C}$, enquanto que a umidade relativa do ar em torno de $87 \%$. Esta espécie foi a mais abundante em área peri-urbana desmatada, e ainda uma das menos capturadas em área de mangue.

O estudo da família Calliphoridae no município de Itaboraí soma-se a muitos outros trabalhos desenvolvidos em outras áreas do território nacional com o propósito de entender o comportamento destes muscóides, podendo-se a partir daí dinamizar-se estratégias mais eficientes em seu controle.

\section{AGRADECIMENTOS}

Ao Instituto Chico Mendes que autorizou as coletas e a Guarda Municipal Ambiental do Município de Itaboraí que nos forneceu suporte logístico durante parte das coletas.

\section{REFERÊNCIAS}

Aguiar-Coelho, V.M. \& E.M.V. Milward-de-Azevedo, 1995. Associação entre larvas de Chrysomya megacephala (Fabricius) e Chrysomya albiceps (Wiedemann), Chrysomya megacephala(Fabricius)eCochliomyiamacelaria(Fabricius) (Calliphoridae, Diptera) sob condições de laboratório. Revista Brasileira de Zoologia, 12: 991- 1000.

Aguiar-Coelho, V.M., M. M. C. Queiroz \& E. M. V. Milwardde-Azevedo, 1995. Associação entre larvas de Cochliomyia macelaria (Fabricius) e Chrysomya albiceps (Wiedemann) (Diptera, Callphoridae) em condições experimentais. Revista Brasileira de Zoologia, 12: 983-990.

Aguiar-Coelho, V.M. \& E.M.V. Milward-de-Azevedo, 1998. Combined rearing of Cochliomyia macellaria (Fabr.) and Chrysomya albiceps (Wied.) (Dipt. Calliphoridae) under laboratory conditions. Journal Applied Entomology, 122: 551-554.

Batista-da-Silva, J.A., H.C. Abádio \& M.M.C. Queiroz, 2009. Miíase humana por Dermatobia hominis (Linneaus Jr.) (Diptera, Cuterebridae) e Cochliomyia hominivorax (Coquerel) (Diptera, Calliphoridae) em Sucessão Parasitária. EntomoBrasilis, 2: 61-63. Disponível em: www.periodico.ebras.bio.br/ojs (07 de abril de 2009)

Baumgartner, D.L. \& B. Greenberg, 1985. Distribution and medical ecology of the blow flies (Diptera: Callphoridae) of Peru. Annals of the Entomological Society of America, 78: 565-587.

d'Almeida, J.M. \& J.R. Almeida, 1998. Nichos Tróficos em dípteros caliptrados, no Rio de Janeiro, RJ. Revisa Brasileira de Biologia, 58: 563-570.

d'Almeida, J.M. \& H.S. Lopes,1983. Sinantropia de dípteros caliptrados (Calliphoridae) no Estado do Rio de Janeiro. Arquivos da Universidade Federal Rural, 6: 39-48.

d'Almeida, J.M., 1994. Ovipositional Substrates Used by Calyptrate Diptera in Tijuca Forest, Rio de Janeiro. Memórias do Instituto Oswaldo Cruz, 89: 261-264.

d'Almeida, J.M., R.P. Mello \& S.M. Oliveira, 1997. Relações tróficas entre dípteros caliptrados (Calliphoridae, Fanniidae, Muscidae e Sarcophagidae), criados em diferentes substratos, no Rio de Janeiro, RJ, Brasil. Entomologia y Vectores, 4: 111121.

Ferreira, M.J.M. \& I.F. Barbola, 1998. Synanthropic taxa, urban, rural and forest habitats, Brazil. Revista Brasileira de Biologia, 58: 203-209.

Ferreira, M.J.M., 1978. Sinantropia de dípteros muscóides de Curitiba, Paraná. I: Calliphoridae. Revista Brasileira de Biologia, 38: 445-454.

Ferreira, M.J.M., 1983. Sinantropia de Calliphoridae (Diptera) em Goiânia, Goiás. Revista Brasileira de Biologia, 43: 199210.

Ferreira, M.J.M., B.B. Santos \& H.F. Cunha, 1995. Flutuação populacional de espécies de Chrysomya Robineau-Desvoidy (Diptera, Calliphoriade) em pomar de Goiânia, Goiás, Brasil. Revista Brasileira de Zoologia, 12: 557-562.

Furlanetto, S.M.P., M.L. C. Campos \& C.M. Hársi, 1984. Microrganismos enteropatogênicos em moscas africanas pertencentes ao gênero Chrysomya (Diptera, Calliphoridae) no Brasil. Revista de Microbiologia, 15: 170-174.

Greenberg, B., 1971. Flies and Disease: Ecology, classification and biotic association. Vol. 1. Princeton Univ. Press., N. J. $856 \mathrm{p}$.

Guimarães, J.H., A.P. Prado \& G.M. Buralli, 1979. Dispersal and distribution of three newly introduced species of Chrysomya (Robineau-Desvoidy) in Brazil (Diptera, Calliphoridae). Revista Brasileira de Entomologia, 23: 245-255.

Guimarães, J.H. ; A.P. Prado \& A.X. Linhares, 1978. Three newly introduced blowfly species in southern Brasil (Diptera: Calliphoridae). Revista Brasileira de Entomologia, 22: 5360

Imbiriba, A.S., D.T. Izutani \& I.T. Milhoreto, 1977. Introdução da Chrysomya chloropyga (Wiedemann, 1818) na região neotropical (Diptera, Calliphoridae). Arquivos de Biologia e Tecnologia, 20: 35-39.

Instituto Nacional de Pesquisas Espaciais, 2008. Disponível em : http://satelite.cptec.inpe.br.

Linhares, A.X., 1981. Synanthropy of Calliphoridae and Sarcophagidae (Diptera) in the city of Campinas, São Paulo, Brazil. Revista Brasileira de Entomologia, 25: 189-215.

Madeira, N.G., E.S. Dias \& C.S. Mascarenas, 1982. Contribuição ao conhecimento da fauna de Calliphoridae (Diptera) Sinantrópicos da Pampulha - Belo Horizonte, Minas Gerais. Revista Brasileira de Entomologia, 26: 137-140.

Mello, R.P. 2003. Chave para identificação das formas adultas das espécies da família Calliphoridae (Diptera, Brachycera, Cyclorrhapha) encontradas no Brasil. Entomologia y Vectores, 10: $255-268$.

Oliveira, V.C., J.M. d'Almeida, M.J.P. SANTOS \& A.SANAVRIA, 1999 . Dinâmica populacional dos dípteros Calliphoridae na Fundação Rio-Zoo, Rio de Janeiro, RJ, Brasil. Entomologia y Vectores, 6: 264-276.

Prado, A.P. \& J.H. Guimarães, 1982. Estado atual de dispersão e dispersão do gênero Chysomya Robineau-Desvoidy na região neotropical (Diptera, Calliphoridae). Revista Brasileira de Entomologia, 26: 225-231.

Queiroz, M.M.C.; P.C. Ribeiro; M.M.O. Cabral; G.E. Moya-Borja; R.P. Mello \& A.N. Norberg, 2005. Miíases Humanas por Cochliomyia hominivorax no Estado do Rio de Janeiro e Suas Conseqüências. Parasitologia Latinoamericana, Santiago, Chile, 6o: 167.

Salviano, R.J.B., R.P. Mello, R.F.S. Santos, L.C.N.H. Beck \& A.Ferreira, 1996. Calliphoridae (Diptera) associated with human corpses in Rio de Janeiro, Brazil. Entomologia y 
Vectores, 3: 145-146.

Santos, B.B., M.J.M. Ferreira \& H.F. Cunha, 1996. Flutuação populacional de alguns Calliphoridae (Diptera) em pomar de Goiânia, Goiás, Brasil. Revista de Agricultura, 71.

Como citar este artigo:

Batista-da-Silva,J.A.,G.E.Moya-Borja\&M.M.C.Queiroz,2010.Ocorrência e Sazonalidade de Muscóides (Diptera, Calliphoridae) de Importância Sanitária no Município de Itaboraí, RJ, Brasil. EntomoBrasilis, 3(1): 16-21. www.periodico.ebras.bio.br/ojs
Recebido em: 29/09/2009

Aceito em: 14/12/2009

$* * * * * * * * * * * *$

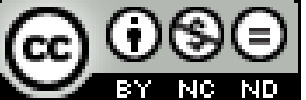

Entomo Brasivis

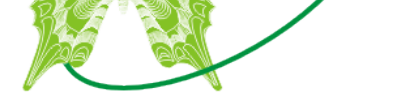

